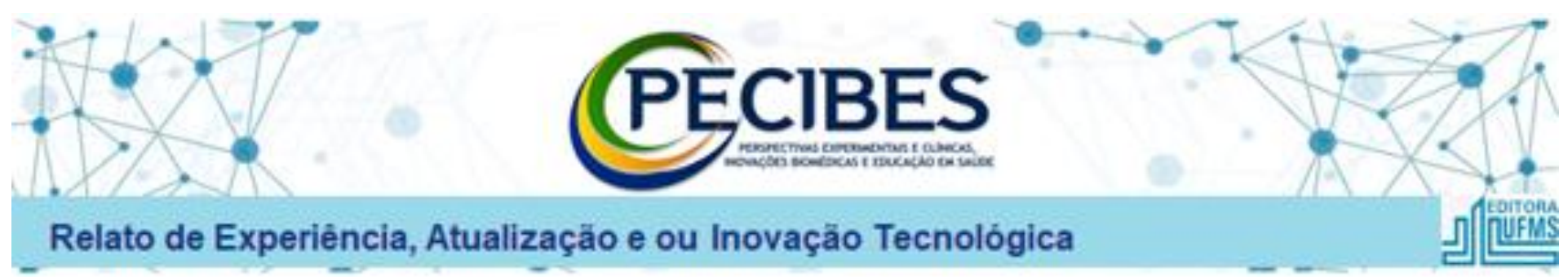

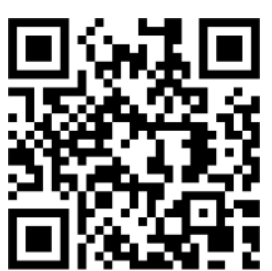

http://www.seer.ufms.br/index.php/peci bes/index

*Autor correspondente: Bertha Lúcia Costa Borges, Hospital Universitário Maria Aparecida Pedrossian Humap-UFMS.

E-mail do autor: bertha.borges@ebserh.g ov.br

Palavras-chave: Tecnologias da informação. Educação Permanente em serviço. equipe multiprofissional.

Key-words: Information technologies. Permanent education in service. multidisciplinary team.

\section{Educação permanente em serviço na linha de cuidado pediátrica com o uso de Tecnologia da Informação e Comunicação}

Permanent in-service education in the pediatric care line using Information and Communication Technology

Aparecida Batista de Sousa Freire ${ }^{1}$, Priscila Cristiane da Silva Mello ${ }^{1}$, Isabela Valerio Guerreiro $^{1}$, Ligia Simões Ferreira ${ }^{1}$, Camilla de Sousa Viana ${ }^{1}$, Andreia Insabralde de Queiroz Cardoso $^{2}$, Bertha Lúcia Costa Borges ${ }^{1}$

\author{
${ }^{1}$ Hospital Universitário Maria Aparecida Pedrossian - Humap - UFMS \\ ${ }^{2}$ Universidade Federal de Mato Grosso do Sul - UFMS
}

\section{Resumo}

Este relato descreve o desenvolvimento de educação permanente em serviço, utilizando as tecnologias da informação como recursos para capacitar uma equipe multiprofissional composta de colaboradores, residentes e graduandos de diferentes especialidades da área da saúde do Hospital Universitário Maria Aparecida Pedrossian - Humap - UFMS a fim de aperfeiçoar os conhecimentos sobre assuntos específicos voltados para a linha de cuidado pediátrico, $\mathrm{O}$ fato do treinamento ser disponibilizado via Ambiente Virtual de aprendizagem e proporcionar atemporalidade de acesso facilitou a consulta do material nos momentos de dúvidas; outro ponto positivo diz respeito à disponibilidade para acesso de possíveis novos funcionários levando em consideração a rotatividade das equipes, proporcionando assim, isonomia no cuidado. Ressalta - se que a produção dos vídeos com a descrição da técnica passo a passo mostrou-se extremamente didática, facilitando a assimilação do conteúdo exibido.

\section{Abstract}

This report requires the development of in-service continuing education, using information technologies as resources to train a multidisciplinary team composed of collaborators, residents and undergraduates from different specialties in the health area of the University Hospital Maria Aparecida Pedrossian Humap - UFMS in order to to improve knowledge on specific subjects related to the pediatric care line, The fact that the treatment is made available via the Virtual Learning Environment and provides timeless access made it easier to consult the material in times of doubt; another positive point concerns the availability for access of possible new employees, taking into account the rotation of the teams, thus providing isonomy in care. It is noteworthy that the production of videos describing the technique step by step is extremely didactic, facilitating the assimilation of the application content. 


\section{Introdução}

A Insuficiência Renal Aguda (IRA) caracteriza-se pela diminuição abrupta da função renal que ocorre por um período de 7 dias ou menos, e a Doença Renal Crônica (DRC) como anormalidades na estrutura ou função renal que persistem por> 90 dias. Em ambas as condições clínicas, a identificação precoce é importante para o manejo adequado e tratamento, com vistas a evitar danos posteriores e mortalidade ${ }^{1}$.

Dentre as opções de Terapia Renal
Substitutiva (TRS) a diálise peritoneal (DP) é a primeira escolha de tratamento para pacientes pediátricos, sendo considerado um método seguro e eficiente para essa população. No entanto, umas das intercorrências mais graves desse método é a ocorrência de peritonite que aumenta os dias de hospitalização, falha na DP e mortalidade. A peritonite está intimamente associada aos erros de técnica e deve ter uma vigilância constante da equipe envolvida no manejo da diálise, bem como estar capacitada e em constante atualização para manutenção dos protocolos estabelecidos ${ }^{2 ; 3}$.

A diálise peritoneal pediátrica ao ser realizada em ambiente hospitalar, deve ser conduzida por uma equipe multidisciplinar de enfermagem e médica especializada em terapia intensiva e nefrologia pediátrica com o objetivo de minimizar intercorrências e fornecer uma terapia de forma adequada e eficiente. Para tal, a equipe multidisciplinar deve receber educação permanente em serviço que reforce a competência e garanta a terapia segura e com qualidade ${ }^{4}$.

$\mathrm{Na}$ área da saúde, a educação permanente em serviço é compreendida como "peça chave" para melhoria de qualidade da vida da população atendida e consequentemente um desafio para profissionais ${ }^{5}$. Neste sentido, entende-se a Educação a Distância como uma modalidade educacional realizada por meio da utilização de tecnologias de informação e comunicação onde educadores e educandos estão em diferentes espaços físicos e de tempo, e pode ser utilizada como grande aliada para educação permanente em ambientes de atendimento à saúde ${ }^{6}$.

As TICs (Tecnologia da Informação e Comunicação) são um conjunto de recursos tecnológicos e computacionais dedicados ao armazenamento, processamento e comunicação da informação de forma unificada, que ultrapassam fronteiras geográficas, culturas e fusos horários, na atualidade

$\mathrm{O}$ acesso à educação, bem como a educação permanente em serviço, não tem tempo ou local fixo; a comunicação de informações armazenadas e acessadas em tempos diferentes passa a ocorrer sem ser perdida a interatividade, de forma veloz ${ }^{7 ; 8}$.

A proposta deste trabalho consistiu no desenvolvimento de educação permanente em serviço, com utilização das TICs como recursos para capacitar a equipe multiprofissional de colaboradores do Hospital Universitário Maria Aparecida Pedrossian - Humap - UFM, de modo a aperfeiçoar os conhecimentos sobre assuntos específicos voltados para a linha de cuidado pediátrico, assim como fortalecer a prática da equipe de saúde no cuidado à família da criança sob à luz dos pressupostos do Cuidado Centrado na Família (CCF).

\section{Material e Métodos}

O Projeto de Educação Permanente foi idealizado pela UCIP - Unidade de Cuidados Intensivos e Semi Intensivos Pediátricos (UTI Pediátrica e Enfermaria Pediátrica) e UASCA - Unidade de Atenção à Saúde da Criança e Adolescente (PAM Pediátrico e Ambulatório de Pediatria) do Humap - UFMS da Rede Ebserh. O público alvo fora os colaboradores lotados nas referidas unidades, bem como os colaboradores de outros setores que demonstrassem interesse sobre o tema. O Projeto foi encaminhado e aprovado pelo Núcleo de Educação Permanente (NEPs) da instituição sob o protocolo SEI 23538.005597/2021-59.

O escopo do treinamento foi produzido com base nas necessidades observadas na avaliação de desempenho dos colaboradores, pela Gestão de Desempenho por Competência (GDC). Foram evidenciadas necessidades relativas aos cuidados específicos em nefrologia pediátrica com enfoque no tratamento dialítico, visto que a instituição é referência para o estado do Mato Grosso do Sul.

Vale ressaltar que foram anteriormente utilizados programas de treinamento voltados para o mesmo público com metodologias convencionais, o que levou a menor abrangência e adesão aos conhecimentos adquiridos, conforme relato da equipe.

Desta forma, houve a busca por novos métodos onde fosse preservada a garantia da continuidade do cuidado aos pacientes, além da abrangência, acessibilidade e o fomento à autonomia dos profissionais de saúde. A educação a distância proporciona por metodologias ativas da aprendizagem e o uso de ferramentas como a plataforma do Ambiente Virtual de Aprendizagem (AVA) as referidas condições.

Foram produzidos materiais audiovisuais no formato de vídeos com a descrição das técnicas com base em evidências científicas. Os mesmos foram elaborados por uma Enfermeira especialista em nefrologia. A gravação e edição foi realizada no Estúdio audiovisual e no laboratório de Habilidades do referido hospital, com materiais de uso contínuo das equipes para proporcionar a vivência o mais próximo possível da realidade.

\section{Resultados}

Foram inscritos nesse treinamento, colaboradores da equipe multiprofissional (médicos preceptores, médicos residentes, enfermeiros, técnicos e auxiliares de enfermagem e fisioterapeutas), além de alunos de graduação de enfermagem, com abrangência para vários setores como CTI Pediátrico, Enfermaria Pediátrica, PAM Pediátrico, Ambulatório de Pediatria, Nefrologia, Neonatologia, entre outros. Embora, do total de inscritos nem todos acessaram a plataforma e/ou concluíram o curso conforme observado na Tabela 1. 
Tabela 1 - Colaboradores inscritos por unidade para capacitação em diálise peritoneal pediátrica.

\begin{tabular}{|l|l|l|l|}
\hline Unidade & Inscritos & $\begin{array}{l}\text { EPS } \\
\text { concluíd } \\
\text { a }\end{array}$ & $\begin{array}{l}\text { EPS não } \\
\text { concluíd } \\
\text { a }\end{array}$ \\
\hline UCIP & 60 & $90 \%(54)$ & $10 \%(6)$ \\
\hline UASCA & 24 & $\begin{array}{l}54,16 \% \\
(13)\end{array}$ & $\begin{array}{l}45,84 \% \\
(11)\end{array}$ \\
\hline OUTROS & 75 & $\begin{array}{l}30,66 \% \\
(23)\end{array}$ & $\begin{array}{l}69,34 \% \\
(52)\end{array}$ \\
\hline TOTAL & 159 & 90 & 69 \\
\hline
\end{tabular}

Tendo em vista o alcance da capacitação para as equipes foi observado uma maior segurança por parte dos profissionais em relação à realização dos procedimentos de diálise peritoneal pediátrica. Visto que os indicadores apontam para menores interrupções da terapia por motivo de dúvida e insegurança diante de intercorrências.

Além disso, a procura pela única profissional especialista da instituição nos momentos de intercorrências diminui significativamente, principalmente fora do horário de trabalho da mesma, fato este que ocorria com frequência. Assim, é evidenciada a maior independência e autonomia da equipe em relação ao procedimento após o treinamento.

$\mathrm{O}$ fato do treinamento ser disponibilizado em modelo EaD e proporcionar atemporalidade de acesso facilitou a consulta do material nos momentos de dúvidas. Outro ponto positivo diz respeito à disponibilidade para acesso de novos funcionários ao considerar a rotatividade das equipes, com isonomia no cuidado. Vale ressaltar que a produção dos vídeos com a descrição das técnicas em formato de passo a passo, demonstra ser extremamente didático e facilita a assimilação do conteúdo apresentado.

\section{Conclusão}

Diante da nova realidade imposta pela pandemia de COVID-19, a Tecnologia da Informação e Comunicação pela modalidade de Ensino à Distância é uma ferramenta essencial para a educação permanente em serviço de saúde e proporciona a chegada do conhecimento a todo o público envolvido, de forma atemporal com o desenvolvimento de maior qualidade técnica da equipe e consequentemente melhora no atendimento das necessidades dos pacientes em tratamento dialítico e suas famílias. Portanto, a escolha do treinamento neste modelo é benéfico e eficaz em relação ao aprendizado e prática na instituição hospitalar.

\section{Agradecimentos}

Agradecemos ao Hospital Universitário Maria
Aparecida Pedrossian - Humap - UFMS, a equipe da Unidade de Websaúde. E aos pequenos pacientes e seus familiares, os quais são o foco de nosso desenvolvimento contínuo.

\section{Referências}

1.Chawla, L., Bellomo, R., Bihorac, A. et al. Acute kidney disease and renal recovery: Consensus report of the Acute Disease Qualit y Initiative (ADQI) Workgroup. Nat Rev Nephrol 13, 241-257 (2017). Disponível em https://doi.org/10.1038/nrneph.2017.2 Acesso em: 29 de nov de 2021.

2.Galasso,

L.; Picca, S.; Dialysis modalities for the management of pediatric acute kidney injury. Pediatr Nephrol. 2020 May;35(5):753-765.

3. Paula M. G. P.; Leite C. M. D. F.; Doença renal crônica na criança. In: PENIDO MGMG, TAVARES MS, KIRSZTAJN GM. (Org.) Nefrologia pediátrica: manual prático. São Paulo: Livraria Balieiro, 2015, p. 279-297.

4.Sanderson, K.

R.; harshman,

A.; Renal replacement therapies for infants an $\mathrm{d}$ children in the ICU. Current Opinion in Ped iatrics: June 2020 - Volume 32 - Issue 3 - p 360-366.

5. Autonomo, Francine Ramos de Oliveira Moura et al. A Preceptoria na Formação Médica e Multiprofissional com Ênfase na Atenção Primária - Análise das Publicações Brasileiras. Rev. bras. educ. med., Rio de Janeiro, v. 39, n. 2, p. 316327, junho $2015 . \quad$ Disponível em: http://www.scielo.br/scielo.php?script=sc i_arttext\&pid=S0100$55022015000200316 \& \operatorname{lng}=$ en\&nrm=iso. Acesso em: 21 set. 2021.

6. Alves, Lucineia. Educação a distância: conceitos e história no Brasil e no mundo. Ver. Bras. Aprendizagem Aberta e a distância - RBAAD, São Paulo, v. 10, n. 1, p. 83-91, Maio, 2011. Disponível em: http://seer.abed.net.br/index.php/RBAAD/arti cle/view/235/113. Acesso em: 21 set. 2021.

7. Moran, J. M. Integração das Tecnologias na Educação. In: Salto para o Futuro. Brasília: Posigraf, 2005.

8. Moran, J. M.; Masetto, M. T.; Behrens, M. A. (Ed.). Novas tecnologias e mediações pedagógicas. 13. ed. São Paulo: Papirus, 2007. 